\title{
Caracterización de Patrones de Variación Morfológica de la Mynthostachys mediante Métodos Estadísticos Multivariante
}

\author{
Doris Gómez ${ }^{1} \quad$ Blanca Martínez ${ }^{1} \quad$ Jorge Condado $^{1}$ \\ dgomezt@unmsm.edu.peGregoria Ramón ${ }^{1}$ \\ Jakov Quinteros $^{2} \quad$ Joaquina Albán ${ }^{3}$
}

\begin{abstract}
Resumen
El objetivo del presente estudio es hacer la caracterización morfológica de Mynthostachys (muña) colectadas en la provincia de Cajatambo, mediante métodos estadísticos multivariantes. El método de Componentes Principal discrimina la existencia de dos especies de Mynthostachys en la comunidad de Cajatambo, las de abundante pubescencia (pubescentes) y las de escasa pubescencia (no pubescentes). La prueba de hipótesis confirma que los vectores de medias basadas en la longitud del pecíolo, largo de la hoja, ancho de la hoja, de las pubescentes y no pubescentes son diferentes.
\end{abstract}

Palabras Clave: Análisis multivariante. Componentes Principales. T2 de Hotelling. Mynthostachys

\begin{abstract}
The main goal of the present study is to make the morphological characterization of the Mynthostachys's (muña) collected in Cajatambo's province, by means of statistical multivariant methods. The main method of Components discriminates against the existence of two Mynthostachys's species(kinds) in Cajatambo's community, those of abundant pubescencia (pubescentes) and those of scanty pubescencia (not pubescentes). The hypothesis test confirms that the vectors of averages based on the length of the petiole, length of the leaf, width of the leaf of the pubescentes and not pubescentes are different.
\end{abstract}

Keywords: Multivariant analysis. Main components. T2 of Hotelling. Mynthostachys

\footnotetext{
${ }^{1}$ UNMSM, Facultad de Ciencias Matemáticas, Lima - Perú.

${ }^{2}$ UNMSM, Instituto Nacional de Salud. Centro Nacional de Salud Intercultural, Lima - Perú.

${ }^{3}$ Depto. de Etnobotánica y Botánica Económica - Museo de Historia Natural.
} 


\section{Introducción}

Minthostachy es una las especies andinas mas fascinantes dentro de las plantas medicinales andinas, puesto que en el mundo de hoy hay una mayor demanda por productos naturales y se pretende alentar el desarrollo de la comunidad andina de Cajatambo, sierra del Departamento de Lima en base a su diversidad y variedades de la especie indicada. Mynthostachys pertenece al reino: Plantae, división: Magnoliophyta, clase: Magnoliopsida, Orden:Labiales, familia Lamiaceae

Brako \& Zaruchi muestra la siguiente distribución de Minthostachys en el Perú.

\begin{tabular}{|l|l|l|}
\hline ESPECIE & ALTITUD & UBICACIÓN \\
\hline $\begin{array}{l}\text { Minthostachys } \\
\text { glabrescens (Bentham) }\end{array}$ & $2500-4000 \mathrm{~m}$ & $\begin{array}{l}\text { Apurimac, Cajamar- } \\
\text { ca, Cuzco, Junín. }\end{array}$ \\
\hline $\begin{array}{l}\text { Minthostachys mollis } \\
\text { (Grisebach) }\end{array}$ & $500-3500 \mathrm{~m}$ & $\begin{array}{l}\text { Amazonas, Are- } \\
\text { quipa, Cajamarca, } \\
\text { Cuzco, Huánuco, } \\
\text { Junín, Lima, La } \\
\text { Libertad, Piura. }\end{array}$ \\
\hline $\begin{array}{l}\text { Minthostachys setosa } \\
\text { (Briquet) Epling }\end{array}$ & $1000-1500 \mathrm{~m}$ & $\begin{array}{l}\text { Puno } \\
\text { Minthostachys tomen- } \\
\text { tosa } \text { (Bentham) }\end{array}$ \\
$2000-3500 \mathrm{~m}$ & $\begin{array}{l}\text { Amazonas, Cajamar- } \\
\text { ca, Cuzco, Huanuco, } \\
\text { Junín, Lima, La Lib- } \\
\text { ertad. }\end{array}$ \\
\hline
\end{tabular}

\section{Materiales y métodos}

El presente estudio se realizó con los datos de Mynthostachys recolectados en la comunidad de Cajatambo del Departamento de Lima.

La Provincia peruana de Cajatambo, es una de las once provincias que conforman el Departamento de Lima, perteneciente a la Región Lima. se ubica en la parte occidental de la cordillera de los andes, entre el departamento de Ancash y las provincias de Oyón y Huaura, a una altura de 3,376 m. con una población de 9,711 habitantes de la que el $58.6 \%$ es rural. Limita al norte con el departamento de Áncash, al este con el departamento de Huánuco, al sur con la provincia de Oyón y al oeste con la provincia de Huaura. Está ubicada en la parte occidental de la cordillera de los Andes.

El muestreo preliminar se realizó entre los meses de Enero a Agosto del año 2004, en un gradiente altitudinal de $2800 \mathrm{~m}$ a $4200 \mathrm{~m}$ en 7 diferentes localidades: Rancas, Astobamba, La Florida, Antay, El Tambo, Cajatambo y Cruzjirca, dentro del ámbito del distrito de Cajatambo. La diversidad y composición florística fueron evaluadas por el método del cuadrante (Caín y Castro 1959; Mostacedo y Fredericksen, 2000), en parcelas de 50m x 2m 
cada una.

Luego en agosto se colectaron las muestras botánicas definitivas de Minthostachys las que fueron evaluadas taxonómicamente en el Herbario de la UNMSM, con el Sistema de Clasificación de Cronquist.

El género Mynthostachys se caracteriza por ser una planta herbácea o sufrutice que se desarrolla entre los 2500 y $4000 \mathrm{msnm}$.

Planta herbácea de $1-1.5 \mathrm{~m}$ de alto, aromática, perenne, cubierta de pelos blandos o velluda (es decir con pelos muy cortos, blandos y rígidos, sensibles al tacto), usualmente creciendo en bordes de chacras de cultivos, bordes de acequias o sobre terrenos bien drenados. Los tallos (ramas), se presentan ampliamente extendidas; erguidas cuando juveniles, y luego se tornan decumbentes (en la madurez), glabrescentes y exfoliantes hacia el extremo subleñoso de la base, curvados y extendidos en forma irregular; pero con pocos o muchas flores pendiendo de éstos.

En la rama principal de cada muestra, las variables morfológicas evaluadas fueron las siguientes:

X1: Longitud del peciolo ( $\mathrm{mm}$ )

$\mathrm{X} 2$ : Largo de la hoja $(\mathrm{mm})$

X3: Ancho del peciolo (mm)

\section{Análisis de Componentes Principales}

El ACP ( Seber,1984; Manly, 1984) es un Método Multivariante de Análisis de datos cuyo objetivo fundamental le permite la reducción de datos de alta dimensión ( $\mathrm{p}>2$ ) en un conjunto de menor dimensión, conservando la máxima variabilidad del problema original, donde las representaciones gráficas y las interpretaciones de las relaciones entre los datos es mucho más sencilla.

El método de obtención consiste en que, con las variables originales $X_{1}, X_{2}, \ldots, X_{p}$ (p > 2) se realizan transformaciones y se encuentran combinaciones lineales de dichas variables, $Y_{1}, Y_{2}, \ldots, Y_{p}$, denominadas Componentes Principales $(\mathrm{CP})$ en el nuevo sistema de menor dimensión, donde estas $\mathrm{CP}$ son variables no correlacionadas y con variancias decrecientes. Es decir:

$$
Y_{i}=\beta_{i 1} X_{1}+\beta_{i 2} X_{2}+\ldots .+\beta_{i p} X_{p} \mathrm{i}=1, \ldots, \mathrm{p}
$$

1. $\operatorname{Cov}\left(Y_{i}, Y_{j}\right)=0 i \neq j i, j=1, \ldots, p$

2. $\operatorname{Var}\left(Y_{i}\right)=\lambda_{i}$

3. $\operatorname{Var}\left(Y_{1}\right) \geq \operatorname{Var}\left(Y_{2}\right) \geq \ldots \ldots \geq \operatorname{Var}\left(Y_{p}\right)$

donde $\lambda_{i}$ es el iésimo autovalor asociado al iésimo autovector $\vec{\beta}_{i}=\left(\beta_{i 1}, \ldots \ldots \beta_{i p}\right)^{\prime}$ de la matriz de covarianzas $\sum$. Sin pérdida de generalidad, para la solución matemática del problema se supone que el vector $\vec{X}=\left(X_{1}, \ldots, X_{p}\right)^{\prime}$ tiene vector de medias $\overrightarrow{0}$ y matriz de covarianzas $\sum$, (Anderson, 1984 ). 


\section{T2 de Hotelling}

Una inquietud interesante que es necesario responder, es realizar el análisis confirmatorio, respondiendo por ejemplo, si existe diferencia significativa entre los vectores de medias de las plantas pubescentes y no pubescentes.

Estadísticasmente el problema se aborda de la siguiente manera: se supone que el vector $\vec{X}=\left(X_{1}, X_{2}, X_{3}\right)$ ' tiene distribución normal multivariante con vector de medias $\vec{\mu}^{(g)}$ y matriz de covarianzas $\sum, g=1,2$, donde $\vec{X}=$ ( longitud del peciolo, largo de la hoja, ancho de la hoja) y se plantea la prueba de hipótesis para los vectores de medias $\quad H_{0}: \vec{\mu}^{1}=\vec{\mu}^{2}$, donde

$$
\begin{gathered}
\vec{u}_{1}=\left[\begin{array}{l}
u_{11} \\
u_{12} \\
u_{13}
\end{array}\right]=\left[\begin{array}{c}
\text { longitud esperada del peciolo, pubescentes } \\
\text { largo de la hoja, pubescentes } \\
\text { ancho de la hoja, pubescentes }
\end{array}\right] \\
\vec{u}_{2}=\left[\begin{array}{l}
u_{21} \\
u_{22} \\
u_{23}
\end{array}\right]=\left[\begin{array}{c}
\text { longitud esperada del peciolo, no pubescentes } \\
\text { largo de la hoja, nó pubescentes } \\
\text { ancho de la hoja, no pubescentes }
\end{array}\right] .
\end{gathered}
$$

Suponiendo que se toman muestras aleatorias independientes de cada uno de los grupos, bajo la hipótesis nula, la estadística $\frac{\left(n_{1}+n_{2}-p-1\right)}{\left(n_{1}+n_{2}-2\right) p} T^{2}$ tiene distribución $\mathrm{F}$ con $\left(p, n_{1}+n_{2}-p-1\right)$ grados de libertad,( Jonshon, 2000; Kraznowsky, 1990; Seber, 1984) donde:

$$
T^{2}=\frac{n_{1} n_{2}}{n_{1}+n_{2}}\left(\vec{X}_{1}-\vec{X}_{2}\right)^{\prime} S^{-1}\left(\vec{X}_{1}-\vec{X}_{2}\right)
$$

$\vec{X}_{1}, \vec{X}_{2}$ : vectores de medias muestrales de las tres variables para plantas pubescentes y no pubescentes respectivamente,

$n_{1}, n_{2}$ : tamaño de las muestras de plantas pubescentes y no pubescentes.

$S_{1} \quad$ y $S_{2}$ :matrices de covarianzas muestrales de plantas pubescentes y no pubescentes $S=\frac{\left(n_{1}-1\right) S_{1}+\left(n_{2}-1\right) S_{2}}{\left(n_{1}+n_{2}-2\right)}$ : Matriz de covarianzas muestral.

\section{Resultados}

Las variables evaluadas en estudio fueron:

$\mathrm{X} 1$ : Longitud del peciolo $(\mathrm{mm})$

$\mathrm{X} 2$ : Largo de la hoja(mm)

X3: Ancho del peciolo(mm)

Los tamaños de las muestras(número de plantas pubescentes y no pubescentes) fueron 51 y 49 respectivamente. El Vector de medias y las matrices de covarianza y correlaciones de las muestras observadas fueron. 


$$
\left[\begin{array}{c}
\bar{X}_{1} \\
\bar{X}_{2} \\
\bar{X}_{3}
\end{array}\right]=\left[\begin{array}{c}
0,8315 \\
3,453 \\
1,9475
\end{array}\right],\left[\begin{array}{lll}
0,257 & 0,212 & 0,177 \\
0,212 & 0,487 & 0,244 \\
0,177 & 0,244 & 0,242
\end{array}\right] \text { y }\left[\begin{array}{ccc}
1 & 0,60 & 0,709 \\
0,60 & 1 & 0,712 \\
0,709 & 0,712 & 1
\end{array}\right] \text { respectiva- }
$$

mente.

Con los autovectores $\left[\begin{array}{ccc}0,5666 & 0,7123 & 0,4143 \\ 0,5675 & -0,7019 & 0,4305 \\ 0,5975 & -0,0088 & -0,8019\end{array}\right]$ asociados a los autovalores $\hat{\lambda}_{1}=$ $2,348, \hat{\lambda}_{2}=0,4$ y $\hat{\lambda}_{3}=0,2514$ se obtuvo las dos primeras componentes principales(CP):

$$
\begin{aligned}
& Y_{1}=1,117 X_{1} \pm 0,8134 X_{2} \pm 1,2144 X_{3}-6,1026 \\
& Y_{2}=1,4044 X_{1}-1,006 X_{2}-0,0179 X_{3} \pm 0,2103
\end{aligned}
$$

Los vectores de medias de las muestras de las plantas con pubescencia abundante y

$$
\begin{aligned}
& \text { pubescencia escasa y la matriz de covarianzas combinada fueron: } \\
& {\left[\begin{array}{c}
\bar{X}_{11} \\
\bar{X}_{12} \\
\bar{X}_{13}
\end{array}\right]=\left[\begin{array}{l}
0,4755 \\
3,2431 \\
1,7363
\end{array}\right]\left[\begin{array}{c}
\bar{X}_{21} \\
\bar{X}_{22} \\
\bar{X}_{23}
\end{array}\right]=\left[\begin{array}{c}
1,2020 \\
3,6713 \\
2,1673
\end{array}\right], S=\left[\begin{array}{ccc}
0,125 & 0,135 & 0,0989 \\
0,135 & 0,445 & 0,200 \\
0,0989 & 0,200 & 0,197
\end{array}\right] \text { re- }}
\end{aligned}
$$

spectivamente.

$$
\vec{X}_{1}-\overrightarrow{\bar{X}}_{2}=\left[\begin{array}{c}
-0,7265 \\
-0,4282 \\
-0,4310
\end{array}\right] S^{-1}=\left[\begin{array}{ccc}
14,2039 & -2,0308 & -5,069 \\
-2,0308 & 4,4234 & -3,4712 \\
-5,0690 & -3,4712 & 11,145
\end{array}\right]
$$

Substituyendo los valores en la correspondiente ecuación se encuentra :

$$
T^{2}=\frac{51(? ?)}{100}(-0,7265,-0,4282,-0,431)^{\prime} S^{-1}(-0,7265,-0,4282,-0,431)=116.4281 \text {, que }
$$

convertida a la estadística $\mathrm{F}$ toma el valor $F_{C}=\frac{(51+49-4)}{(100-2) 3} T^{2}=114,05$

\section{Análisis y discusión}

Las dos primeras componentes principales

$$
\begin{aligned}
& Y_{1}=1,117 X_{1} \pm 0,8134 X_{2} \pm 1,2144 X_{3}-6,1026 \\
& Y_{2}=1,4044 X_{1}-1,006 X_{2}-0,0179 X_{3} \pm 0,2103
\end{aligned}
$$

retienen el $91.6 \%$ de la variabilidad de las variables originales. Reemplazando las puntuaciones originales de cada indivíduo en cada una de las combinaciones lineales $Y_{1}$ y $Y_{2}$ se obtiene las puntuaciones en la Componentes Principales para cada individuo. Dicha información presentada en el Gráfico No 1, indica claramente la presencia de dos tipos de Mintosttachy en el Distrito de Cajatambo. En el Laboratorio de Etnobotánica han caracterizado dichas especies como Pubescentes (pubescencia abundante) y No Pubescentes (pubescencia escasa). 
Gráfico No 1: Plantas de Mynthostachy proyectadas en las dos primeras Componentes Principales

Dicho resultado se confirma al observar que las correlaciones entre la primera componente principal y las variables originales son altas.

Cuadro No 1: Matriz de Correlaciones

\begin{tabular}{|l|l|l|l|}
\hline Originales & Y1 & Y2 & Y3 \\
\hline X1: Longitud del peciolo & 0.8683 & 0.4505 & 0.2077 \\
\hline X2: Largo de la hoja & 0.8697 & -0.4439 & 0.2158 \\
\hline X3: Ancho de la hoja & 0.9156 & -0.0056 & -0.4020 \\
\hline
\end{tabular}

La matriz de correlaciones entre las variables originales y las componentes principales nos indica claramente que tenemos dos tipos de Mynthostachys. Dicha información presentada en el Gráfico No 1, indica claramente la presencia de dos tipos de Mintosttachy en el Distrito de Cajatambo.

El valor de $T^{2}=116,4281$ es transformado a la estadística $\mathrm{F}$ y toma el valor $F_{C}=114,05$ , que luego es comparado con el cuantil de la distribución F con 3 y 97 grados de libertad con una probabilidad de $0.95, F(3,97,0,05)=2,7$, consecuentemente, la prueba de hipótesis es significativa al $5 \%$. Por tanto, podemos concluir que existe evidencia suficiente para afirmar que los vectores de medias de las plantas con abundante pubescencia y escasa pubescencia son diferentes.

\section{Conclusiones}

El Análisis de Componentes Principales muestra que son dos los tipos de Mynthostachys en el Distrito de Cajatambo de la Provincia de Cajatambo del Departamento de Lima.

El análisis de comparación de medias de los dos tipos de Mynthostachys confirma la existencia de diferencias estadísticamente significativas entre dichos vectores de medias.

\section{Referencias}

[1] ANDERSON T.W. An introduction to multivariate analysis. John Wiley (1984)

[2] ALVAN, C.J. Un registro de datos etnobotánicos . Boletín de Lima, No 39, (1985).

[3] CAIN y CASTRO Manual of Vegetation Analysis. Harper and Brothers Publishers, New York (1959). 
[4] CRIDCI J. Introducción a la teoría y práctica de la taxonomía numérica. Serie de Biología. OEA (1983).

[5] MANLY B. multivariate statistical methods. Chapman Hall (1986).

[6] MOSTACEDO B. FREDERICKSEN Manual de métodos básicos de muestreo y análisis en ecología vegetal. Bolford (2000)

[7] JOHNSON Métodos multivariantes aplicados al análisis de datos (2000).

[8] KRZANOWSKI Principles of multivariate análisis. Oxford statistical science series (1990).

[9] SEBER Multivariate observations. John Wiley (1984). 